\title{
Transfer of training from introductory computer courses is highly specific ... and negative!
}

\author{
KIRK H. SMITH, DIETER ZIRKLER, and BARBEE T. MYNATT \\ Bowling Green State University, Bowling Green, Ohio
}

\begin{abstract}
Two groups of students in an introductory computer course learned either BASIC programming or the use of applications software. Students in the programming group learned to use a simple line editor to edit their programs, whereas students in the applications group learned to use a sophisticated cursor-oriented word processor. When both groups were tested on a simulated line-oriented text editor, there were no differences in overall performance, although both groups performed more poorly than did a group of introductory psychology students with no previous computer background. Different error patterns in the two groups for different commands suggest specific kinds of negative transfer due to their prior experiences. These results call into question the assumption that training students in one computer skill facilitates transfer to other situations.
\end{abstract}

The proliferation of microcomputers in institutions of higher education has increased interest in educational programs to introduce students to computers. Traditionally, such courses have included a hands-on component in which students learn how to program, usually in a higher level language such as BASIC. Originally, this content was justified by the fact that most of the applications that students might expect to encounter required some programming. Although the preponderance of microcomputers on today's market still include a BASIC interpreter in the software bundle, the practical need is decreasing in importance. For a student who expects to use readily available applications programs such as word processors, spreadsheets, and the like, there is little immediate justification for mastery of programming in any form.

The absence of a practical need for programming experience raises questions about its contribution to understanding and appreciating the capabilities and limitations of computers. One appealing alternative to hands-on experience in programming is training in the use of one or more application packages of immediate academic relevance. For example, given the emphasis placed on writing at the college level, the hands-on component of an introductory computer course might include intensive

The research described in this report was made possible by the Sanyo Business Systems Corporation of Moonachie, NJ, which lent Bowling Green State University 10 complete microcomputer systems, including software and printers, during the academic year 1983-1984. We especially want to thank Arthur Shebar, National Sales Manager, for arranging the details of the loan. Many other individuals contributed to the computer education project of which this experiment was a part. We thank especially Steve Zirkel, who assisted in collecting the data, Richard L. Conrad and Charles Schultz, who arranged for us to use IBM PCs belonging to the University's Computer Services for the 2week testing period, and Ron Milos, who, as manager of the Dealer Support Department of Sanyo Business Systems' computer division, enthusiastically supported the project. The authors' mailing address is: Department of Psychology, Bowling Green State University, Bowling Green, $\mathrm{OH} 43403$. training in the use of word processors. Such an experience would give students a glimpse of computer capabilities and limitations and help alleviate any fear they might have about working with computers. Moreover, a properly managed program of this kind would provide a resource that they could use immediately in preparing reports and term papers.

Once a viable alternative to instruction in programming is presented, questions arise about the cognitive products of the two approaches. A question of considerable practical interest is which one of these alternative instructional approaches makes it easier for students to learn how to use new computer programs. Today's students will almost certainly be faced with mastering the use of different hardware and software from that now available for instruction. The advantages of acquiring an immediately useful skill might be offset by negative transfer in learning to use another computer system.

The present study was designed to investigate what is learned in the hands-on component of introductory computer courses. Specifically, we compared the two educational strategies, programming and applications. One group of students was given instructional materials and assignments that required them to learn the fundamentals of computer programming by creating and successfully running programs in BASIC. The programming was carried out on Apple II + or IIe computers. Another group received materials and assignments that required that they learn how to use a word processing program and, to a lesser extent, a spreadsheet package. Training on applications software occurred on Sanyo MBC- 1150 computers (using the $\mathrm{Z80A}$ processor to run the $\mathrm{CP} / \mathrm{M} 2.2$ operating system). Both groups attended weekly lectures and were required to read common assignments in a computer literacy textbook.

Midway through the semester, students participated in four hands-on exercises designed to explore how well they could generalize what they had learned to other types of 
computer applications. In this paper, we report on students' performance on a simulated text editor program designed to measure the extent to which previous experience in writing BASIC programs or in using specific applications software transfers to a new situation. In order to determine the absolute, as well as the relative, effect of these two types of hands-on experience with computers, we also tested a group of introductory psychology students who had had no previous computer experience.

The experimental program, Recipe Editor, was designed to simulate for the user the process of learning to use a simplified command language by translating syntactic formulas and examples into the required commands. The command language was modeled on a mainframe editing system, which was used chiefly by a small population of sophisticated computer users. It features very concise, abstract commands with a rigorous syntax. In a full implementation of such a system, these commands would have great generality and power. However, Recipe Editor is a simulation, not a functional text editor. The program did not permit the user to deviate from the sequence of editing operations specified in the instructions. Although this restriction was somewhat artificial, it prevented our subjects from becoming hopelessly entangled in chains of errors. The program was implemented on the IBM PC-hardware that was new to all three groups.

The Recipe Editor is a line editor and combines functions from both the Applesoft BASIC editor and WordStar, the word processing program one of the groups of students learned. The Recipe Editor requires the user to issue complete one-line commands, including a line number, in the manner of the Applesoft editor. In WordStar, text is treated as continuous, and a cursor, instead of a line number, is used to select the portion of text to be manipulated. The Recipe Editor contains a richer set of operations than the limited options available in the Applesoft editor. The Recipe Editor contains instructions to center text on a line and to insert and delete characters and words. These functions are similar to some of many features found in WordStar.

The educational philosophy motivating computer literacy courses implies that both groups of students in the computer course will have an advantage over students with no previous experience because they will have a better general understanding and appreciation of computers and their operation. It is also assumed that students should have overcome any initial fear of computers after several weeks of working with the machine. Further predictions can be derived from a consideration of the knowledge gained by the two computer groups.

The applications group might be expected to have a much more complete conception of how computer text editors work. Recipe Editor and WordStar are both text editors, and therefore, in overall function and purpose, they are similar enough that some general positive transfer might be expected for the applications group. On the other hand, this group's knowledge was very specific. To the extent that the Recipe Editor required them to learn to perform the same editing functions in a different way, negative transfer might also be expected.

The conceptual contributions of studying programming are a matter of speculation, but one possibility is that the students exposed to hands-on experience with writing and debugging programs might acquire a more general understanding of computer programs of all kinds. Thus, the programming group might display an overall advantage in learning to use the Recipe Editor.

A plausible prediction is that each group has an advantage in learning those operations that are already familiar from the setting in which they had worked. Thus, the applications group might be expected to perform well on inserting and deleting characters (frequently used, singlekeystroke operations in WordStar) and more poorly on inserting lines (which has no true counterpart in WordStar). By contrast, the group that studied programming would be expected to show positive transfer to operations involving line insertion and deletion, because these are frequently used operations in writing and editing BASIC programs.

An alternative hypothesis, derived from interference theory, makes just the opposite prediction. Students who have learned a particular way of carrying out a certain function, such as line insertion, might have difficulty understanding and learning how to perform this or a similar function on a different system. Extensive users of computer application packages often complain about this phenomenon as they move between several different text editors, statistical packages, or whatever. The problem emerges with less sophisticated users when, for example, a word processor, a spreadsheet, and a database management system perform certain functions (such as moving the cursor) in different ways. Thus, a case can be made for predicting negative as well as positive transfer.

\section{METHOD}

\section{Subjects}

The subjects were 36 undergraduate students enrolled in a computer science course entitled "Computer Basics" and 9 enrolled in an introductory psychology course. The latter students received course credit for participation. Enrollment in the computer course was initially limited to students with a minimum of sophomore standing, but during the period of drop/add prior to the beginning of the semester, this restriction was not consistently observed. Students were assigned randomly to one of the two groups: programming, using BASIC on the Apple II + or Ile, and applications, using WordStar and CalcStar on the Sanyo MBC-1150.

\section{Training}

Students assigned to learn BASIC programming were given a detailed set of handouts that outlined weekly study assignments in the self-teaching text, BASIC for the Apple II (Brown, Finkel, \& Albrecht, 1982), a set of 
programming assignments, and administrative information about campus computer facilities and the collection and grading of assignments. These students received a 30to 45-min demonstration of computer-aided instruction or computer graphics on the Apple II and participated in an initial session of 60 to $90 \mathrm{~min}$ in which they were shown how to use the hardware and operating system to enter and run programs in BASIC. At this time, their personal disks were initialized. After these preliminaries, students in this group worked individually. They were free to work on assignments any time computers were available in a number of computer laboratories. There was an optional 1-h lecture and help session each week. The initial handout information sheet explained how the student could obtain help or tutoring from special programming consultants as well as from one of the course instructors. However, in most cases, obtaining help required that the students initiate a search and often involved a delay.

Students learning how to use applications programs were assigned to sections of from five to seven students that met for $\mathbf{2} \mathrm{h}$ twice a week in a special laboratory housing the Sanyo MBC-1150 computer systems. Each section was staffed by a graduate or undergraduate monitor who was familiar with the systems and the assignments. Assignments for the first 2 weeks were described in an initial handout; thereafter, students obtained handouts describing each week's assignments from the section monitor. Assignment sheets described the learning objective for each week and presented a detailed description of what was expected and how it would be graded. Suggested reading assignments and reference materials in the WordStar Training Guide (MicroPro International Corporation, 1981), the CalcStar User's Manual (MicroPro International Corporation, 1982), and the user's guide for the Sanyo MBC-1100 Series computers were also listed.

An important difference between the two groups was the fact that students in the applications group were expected to attend regular laboratory sessions where they knew a computer would always be available for them and where the monitor was available to answer questions and help with problems. Both groups attended the same series of seven 50-min lectures on computers and how they work. This included a very elementary introduction to programming concepts. Assigned readings (from Spencer, 1983) covered the same factual and conceptual material as the lectures. All students were tested during a 2-week period of diverse activity (a midterm test, a hands-on test of either BASIC programming or specific applications skills, and three other exercises similar to the one described in the present paper).

\section{Software}

The Recipe Editor was written in UCSD Pascal, and was designed to present subjects with three recipes and to require nine corrections in each recipe. Each correction corresponded to the use of a specific editor command. The nine commands were (1) moving from one recipe (screen) to another, (2) centering a line, (3) capitalizing a character, (4) deleting a character, (5) deleting a word, (6) deleting a line, (7) inserting a character, (8) inserting a word, and (9) inserting a line. Subjects were required to complete the corrections in a specific order. Each correction had only one correct solution. Alternative solutions were not accepted by the editor and were considered errors. Figure 1 illustrates the format of the Recipe Editor screen. Commands were entered by subjects on the Command line of the editor screen. The editor responded to incorrect commands by printing "Wrong CommandTry Again' ' on the Status line. Correct responses caused the editor to execute the command, and the effect of the command was printed on the Status line. The order of the corrections was randomized within each recipe.

\section{Materials}

An instruction manual was developed to describe the experimental task to the subjects. The instruction manual first described the format of all nine Recipe Editor commands. Figure 2 illustrates the format of the nine commands. Special keys on the computer keyboard were also described. Next, the order of corrections was listed for each recipe. Each instruction described the required correction and the line number of the correction. Finally, a reference section provided examples of how each command could be used and the effect of the commands on text.

\section{Procedure}

Subjects were first assigned to one of two IBM PCs. Both microcomputers were identical and were equipped with identical IBM monochrome monitors. Subjects were seated in front of the computer. The monitor screen was turned off at this point. Subjects were told that they would be using an editor to correct several recipes. They were then given the instruction manual and instructed to read the manual at their own pace. When subjects had finished reading the manual, the experimenter asked them if they had any questions. After questions had been answered, the experimenter turned on the monitor screen and recorded the start time. Subjects were then instructed to begin. They were required to correct three recipes. Sub-

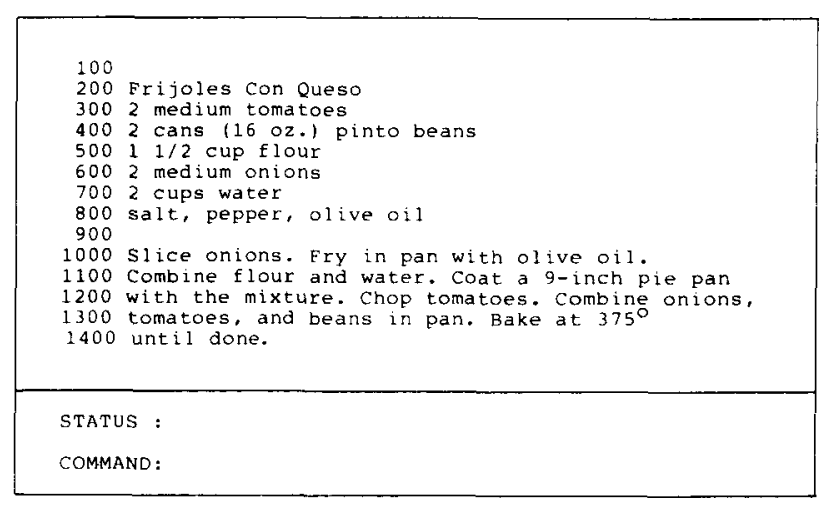

Figure 1. The recipe editor screen. 


\begin{tabular}{|c|c|}
\hline Command & Format \\
\hline Select recipes & REC [recipe \#] \\
\hline Center text on line & C lline \#] \\
\hline Capitalize character & $\begin{array}{l}\text { CAP [line \#]*word to be } \\
\text { capitalized*first letcer }\end{array}$ \\
\hline Delete character & $\begin{array}{l}\left.\text { D }[\text { line \# }]^{*} \text { o }\right] d \text { spelling } \\
\text { of word*new spelling }\end{array}$ \\
\hline Delete word & D [line \#]*word* \\
\hline Delete line & D [1 ine \#]** \\
\hline Insert character & $\begin{array}{l}\text { I [ } 1 \text { ine \#]*unique string } \\
{ }^{\star} \text { new character }\end{array}$ \\
\hline Insert word & $\begin{array}{l}\text { 1 [ line \#]*word before } \\
\text { insertion*new words }\end{array}$ \\
\hline Insert line & I lline \#]** \\
\hline
\end{tabular}

Figure 2. The formats of the nine Recipe Editor commands as they appeared in the subjects' instruction manual.

jects were permitted and encouraged to refer to the instruction manual. Although the experimenter answered hardware-oriented questions (e.g., "Where is the RETURN key?'), software-oriented questions (e.g., ' 'How do I delete a word?'”) were not answered. The experimenter responded to software-related questions by referring the subjects to the instruction manual. When subjects had completed the experimental task, the end time was recorded. Subjects were then debriefed.

\section{RESULTS AND DISCUSSION}

The number of errors made by each subject on each of the nine types of corrections was recorded. An error was defined as any command line entered by the subject that failed to execute a required correction. The amount of time, in minutes, taken by a subject to complete the experimental task was also recorded. The time required for subjects to read the instruction manual was not included in this measure. A multivariate analysis of variance of the total number of errors and the time to completion using Wilk's criterion indicated that there were differences among the three groups $[\mathrm{F}(4,82)=2.80$, $\mathrm{p}=.03]$. The means are shown in Table 1 .

Separate univariate analyses of variance were conducted on completion time and number of errors using two planned comparisons, one that contrasted the group with no previous experience with the two computer literacy groups and a second that contrasted applications with programming. (All statistical tests reported in this paper were conducted at the $<.05$ level of significance.) The group with no computer experience took significantly less time than the two computer literacy groups $[\mathrm{F}(1,42)=$ $6.81, \mathrm{MSe}=218.92 \mathrm{~J}$, but the latter two groups did not differ significantly $(F<1.00)$. Similarly, the group with no computer experience made fewer errors than the other two groups $[\mathrm{F}(1,42)=9.74, \mathrm{MSe}=105.02]$, but the latter two groups did not differ from each other $[\mathrm{F}(1,42)$ $=2.49]$.
The failure to find a difference between the two computer literacy groups is consistent with other similar measurements on these individuals. For example, there was no difference in their performance on the common items of the midterm examination. Similarly, these groups did not differ in overall performance on an exercise that required that they learn to use a computerized concordance for the King James Bible (Mynatt, Smith, Kamouri, \& Tykodi, 1985). Taken together, this pattern of results suggests that there are probably no major intellectual or practical effects on general background of two quite different types of hands-on supplements to introductory computer courses. Obviously, this conclusion needs to be tested further. It may be that procedural differences, such as the use of a self-teaching text with limited class meetings for the programming group, may have offset what would have been an advantage for this group, although this seems unlikely.

A univariate analysis of variance was performed on the number of errors each subject committed for each command and recipe. (No time data were available for individual commands.) Because all subjects were exposed to the three recipes in the same order, the recipe factor was confounded with trials on each command. (This factor will be referred to as "trials," because this has a more general and interpretable meaning.) The analysis consisted of a 2 (training groups) $\times 9$ (commands) $\times 3$ (trials) design.

As expected from the multivariate analysis, the three groups differed significantly in overall error rate. In addition, there were significant main effects due to commands $[\mathrm{F}(8,336)=7.46, \mathrm{MSe}=3.80]$ and trials $[\mathrm{F}(2,84)$ $=13.61, \mathrm{MSe}=4.89]$, as well as several significant interactions and a significant three-way interaction $[\mathrm{F}(32,672)=2.21, \mathrm{MSe}=4.06]$. The latter can be best understood in the context of the interactions between commands and groups $[\mathrm{F}(16,336)=2.42, \mathrm{MSe}=3.80]$ and between commands and trials $[\mathrm{F}(16,672)=6.36$, MSe $=$ 4.06].

Table 2 displays the interaction between commands and groups. The most striking feature of these data is the differences in the patterns of difficulty for the three groups. The most difficult command overall was word insertion, and the two computer literacy groups did not differ appreciably on this command. However, the most difficult command for the programming group was line deletion. On both line deletion and line insertion, the programming group made more errors than did the ap-

Table 1

Mean Number of Errors and Completion Time (in Minutes) for Groups With Different Backgrounds in Computer Use

\begin{tabular}{lccr}
\hline & \multicolumn{3}{c}{ Background } \\
\cline { 2 - 4 } Command & Applications & Programming & None \\
\hline Mean number of errors & 18.8 & 22.2 & 8.9 \\
Mean completion time & 46.4 & 49.0 & 33.3 \\
\hline
\end{tabular}

Note-There were 18 students each in the applications and programming groups, and 9 in the group with no computer background (None). 
Table 2

Mean Number of Errors by Command for Groups With Different Backgrounds in Computer Use

\begin{tabular}{lccc}
\hline & \multicolumn{3}{c}{ Background } \\
\cline { 2 - 4 } \multicolumn{1}{c}{ Command } & Applications & Programming & None \\
\hline Select recipes & .06 & .11 & .07 \\
Center text on line & .65 & .17 & .07 \\
Capitalize a character & .43 & .11 & .33 \\
Delete character & .81 & .44 & .22 \\
Delete word & .28 & .38 & .19 \\
Delete line & .54 & 2.35 & .15 \\
Insert character & .70 & .41 & .33 \\
Insert word & 1.98 & 1.85 & .85 \\
Insert line & .82 & 1.56 & .74 \\
\hline
\end{tabular}

Note-There were 18 students each in the applications and programming groups, and 9 in the group with no computer background (None).

plications group. These are precisely the commands on which the programming group was expected to excel, because the Recipe Editor's greatest similarity to the concepts inherent in the Applesoft BASIC editor lies in its use of line numbers in inserting and deleting lines.

Of the six less difficult commands, it was expected that the applications group would have an advantage on commands that manipulated single characters, due to WordStar's character-oriented operation. Operations on single characters in WordStar require one or, at the most, two keystrokes once the cursor has been placed. Likewise, centering text on a line can be done with a single keystroke (or with two) in WordStar, and should have been a familiar concept to the applications students. Instead, the applications group made more errors on these commands than did the programming group. The groups did not differ on the word-deletion command or on the easiest command, that for selecting the next recipe.

Negative transfer most parsimoniously describes both the overall performance of the groups and the pattern of errors across commands. Students with some previous computer experience had the greatest difficulty in learning to perform familiar operations with new procedures. The considerable practice of the students who learned how to manipulate characters in WordStar appears to have left a set of biases about how computer systems manipulate characters. Faced with a conceptually different technique for inserting and deleting characters, these students encountered difficulties.

The students who studied BASIC programming seem to have acquired an unintended lesson. They inadvertently learned something about editing in general and line manipulation in particular. Their errors are particularly interesting because the editing facilities of Applesoft BASIC are quite primitive. We doubt that most instructors of introductory programming courses have considered the impact of the editing facilities of the programming language(s) on their students' appreciation of computer capabilities and limitations. Perhaps students learning to program with poor editors infer that editing text in computer systems is difficult and unwieldy-exactly the opposite of what a student would gather from learning to use any reasonable word processor.
Tukey's studentized range test was used to assess the effect of trials (hsd $=.41$ ). The bulk of the errors occurred on the first trial, on which the mean per command was 1.25 . The latter was significantly different from the mean of .45 on the second trial and .31 on the third trial. Trials 2 and 3 did not differ. The interaction between commands and trials consisted principally of deviant patterns of errors over trials for two commands, a very high error rate on the second trial for the insert-line command and an elevated error rate on the third trial for the deletecharacter command. These can be understood in terms of the three-way interaction. Figure 3 presents error rates for the applications and programming groups by trials for four commands, the two deviant ones and two commands that are representative of the overall pattern of rapid decline from Trial 1 to Trial 2. (The error rate for the group with no previous computer background was so low that there was little meaningful variation across trials.)

The line-deletion command for the applications group is typical of the overall pattern, as is the character-deletion command for this group. The special difficulty of this command for the programming group occurred on Trial 1 and was resolved by the Trial 2 . Line insertion was also more difficult for the programming group, but the difficulty was manifest on Trial 2 . The explanation lies in the fact that, on Trials 1 and 3, subjects were required
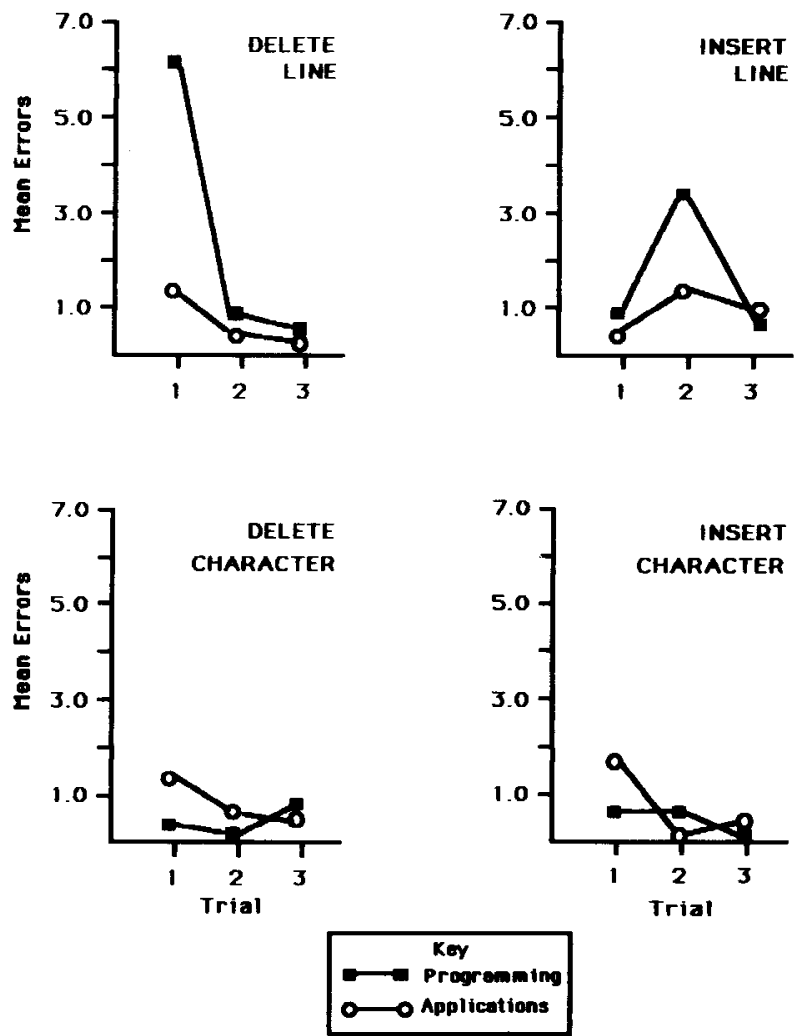

Figure 3. Mean number of errors by trials for two groups of students in a computer literacy course for four representative editing commands. 
to insert a blank line, whereas, on Trial 2, the line to be inserted contained text. Note that both groups had more difficulty on Trial 2 . The patterns are less clear for the character-manipulation commands. The applications group had relatively more difficulty with these commands, but the errors tended to occur on the first trial. This was also the case with the text-centering command.

Although these results suggest that the negative transfer was short-lived, it is important to keep in mind that the participants in this study were not using a functional line editor. The Recipe Editor notified the users when they had made a mistake instead of carrying out the entered command. Such feedback is potentially useful, of course, but with learners it is just as likely to create a new situation that is even more difficult to deal with than the original one. Thus, if anything, our simulated editor was probably easier to use than a functional text editor. And the number of errors observed may well be an underestimate of the difficulty people have in learning to use new systems.

The negative transfer observed in this experiment may be limited to individuals who have learned one system for performing a set of functions and are then required to learn a new one to perform the same functions. The phenomenon may also be limited to software that is relatively difficult to master. Students from both the applications and programming groups performed better than computernaive subjects with the computerized Bible concordance, a program written specifically for users with limited computer background (Smith et al., 1984).

\section{SUMMARY AND CONCLUSIONS}

The effects of two kinds of hands-on experience with computers were not apparent in global measures of performance with the simulated editor. However, there was evidence for transfer of specific knowledge and skills, but not in the direction predicted. Experience with certain computer operations and processes as an adjunct to a computer course made it more difficult for students to carry out similar tasks with new software, not easier, as one might expect. Far from providing a general background in computation, learning to program in BASIC appears to have narrowed the students' capacity to work with new software that differed in critical ways.

These instances of negative transfer were surprisingly specific. Having learned one very limited way to manipulate lines on a computer monitor, students who studied programming seem to have developed blocks to mastering more powerful techniques of this kind. Similarly, students who had mastered one way of manipulating characters in the process of learning how to use a word processor that focused attention on characters seemed to develop a temporary blindness to an alternative method of manipulation that could be quite powerful in other applications.

In general, the results seem to point to the overlooked and unintended specificity of learning that occurs in the hands-on component of introductory computer courses. If the present results can be replicated and converging research designs corroborate the conclusions, it will be necessary to consider what kinds of experiences are necessary for inculcating a broad set of computer capabilities.

\section{REFERENCES}

Brown, J. R., Finkel, L., \& Albrecht, R. L. (1982). BASIC for the Apple II: A self-teaching guide. New York: Wiley.

Micropro International Corporation. (1982). CalcStar user's manual. San Rafael, CA: Author.

MicroPro International Corporation. (1981). WordStar training guide. San Rafael, CA: Author.

MYNATT, B. T., Smith, K. H., Kamour, A. L., \& Tykod, T. A. (1985). Which way to computer literacy, programming or applications experience? Manuscript submitted for publication. SPENCER, D. D. (1983). An introduction to computers: Developing computer literacy. Columbus, $\mathrm{OH}$ : Merrill. 